\title{
An Efficient System for Detecting Forest Fire using Spatial Data
}

\author{
L. Ramesh and E. Dinesh \\ Department of Electronics and Communication Engineering, M. Kumarasamy College of Engineering, \\ Karur-639113,Tamil Nadu, India; rameshl.ece@mkce.ac.in, dineshe.ece@mkce.ac.in
}

\begin{abstract}
Objectives: To detect fire in forest areas using spatial information. Methods/Statistical Analysis: The spatial data help in finding the areas powerless against woodland fire with the guide of Image handling. The Fuzzy C Mean algorithm is employed in design of the effective system. Initially, the images with the presence of fires are converted from RGB to XYZ color space. These images are clustered using FCM algorithm which locates the regions of fire. Findings: At first, the pictures with the nearness of flames are changed over from RGB to XYZ shading space. Thus, the XYZ shading space estimations of pixels which are bunched for recognizing the nearness of flames. Subsequently our composed successful framework will help the general population in reconnaissance to recognize timberland fires and to take suitable activities. Application/Improvements: This system is designed and produces the effective result for the people in surveillance of fire areas.
\end{abstract}

Keywords: CIE, Fuzzy C Means Clustering, K Means Clustering, XYZ

\section{Introduction}

The terms examination and amalgamation start from built up Greek where they mean independently "to disassemble" and "to collect". These terms are used as a piece of sensible controls from science and reason to economy and mind research to mean practically identical investigative approach. Examination is described as the technique by which we isolate an academic or extensive whole into parts or sections. Blend is described as the reverse: to join detach segments or parts with a particular true objective to outline a clear total ${ }^{\perp}$. Structures examination authorities apply theory to the examination of the systems required to shape a general picture. A frameworks investigation identified with open choices is frequently alluded to as a strategy examination (in the United States the terms are utilized conversely). A frameworks ${ }^{1}$ examination that focuses on correlation and positioning of options on premise of their referred to qualities is alluded to as choice investigation 2 . Systems examination usually has some mix of the going with: Recognizing confirmation and re-ID of targets, confinement and elective courses of action; examination of the probable results of the decisions to the extent costs, points of interest and risks; presentation of the results in a comparable structure so the pioneer can settle on a good choice from among the decisions. The average utilization of frameworks examination is to direct choices on issues, for example, national or corporate plans and projects, asset utilize and insurance approaches, innovative work $^{2}$ in innovation, provincial and urban advancement, instructive frameworks and other social administrations. Obviously, the nature of these issues requires an interdisciplinary approach

\section{Methodologies and Algorithms}

The existing system in forest fire detection is the integrated false alarm system ${ }^{3}$. The existing system accessing of information is very slow and it required a lot of time to organize the data for a consolidated report. The existing system process and method of organizing and maintenances are studied with appropriate details. Forest flames are a worldwide concern causing a considerable measure 
of harm and adding to the decay of the Earth environment, especially to the dangerous atmospheric deviation. In this existing system, as shown in Figure 1, the problem of detecting forest fires ${ }^{3}$ is done manually. The forest fires are highly connected with the problem of deforestation. Because of the forest fires, several hundred million hectares of forest and other vegetation are devastated each year. In past several years some research efforts have been concentrated on detecting forest fires and locating the regions.

\subsection{Drawbacks}

There has not been a good way to resolve the problem of detecting forest fires because it is hard to tackle the problem such as finding the region, influence of luminance and intensity values and so on. It was overcome by using the proposed system. The dependability of integrated location frameworks is as yet a critical issue in the domain. The most imperative downsides of existing programmed backwoods fire identification frameworks are high false caution rate, low practical scope.

Bunching examination ${ }^{4}$ has been a rising exploration issue in information mining due its assortment of utilizations. With the appearance of numerous information bunching calculations in the current couple of years and its broad use in wide assortment of uses, including picture handling, computational science, versatile correspondence, prescription and financial aspects, has prompt the prevalence of this calculations set into homogeneous gatherings in light of given highlights to such an extent that similar items are kept in a gathering while disparate articles are in various gatherings. It is the most crucial unsupervised learning issue. It oversees finding structure in a gathering of unlabeled data.

\subsection{Requirements of Clustering}

- Scalability information must be versatile else we may misunderstand the outcome. It must have the capacity to manage diverse kinds of characteristics. Likewise ready to discover grouped information with the discretionary shape. It must be obtuse to commotion and exceptions.

- Decipher capacity and usability - Result got must be interpretable and usable so most noteworthy finding out about the information parameters can be obtained. It must have the ability to oversee educational accumulation of high dimensionality.

\subsection{Clustering Algorithms}

Clustering methods $s^{4}$ can be classified into two types:

- Unsupervised method

- Unsupervised non-linear method

\subsubsection{Unsupervised Method}

- K-means clustering algorithm.

- Fuzzy C-means clustering algorithm.

- Hierarchical clustering algorithm.

- Gaussian (EM) clustering algorithm.

- Quality threshold clustering algorithm.

\subsubsection{Unsupervised Non-Linear Method}

- MST based clustering algorithm.

- Kernel K-means clustering algorithm.

- Distance Measure.

A vital segment of a bunching calculation is the separation measure between information focuses. On the off chance that the parts of the information example vectors are all in the same physical units then it is conceivable that the basic Euclidean separation metric is adequate to effectively gather comparative information cases. In any case, even for this situation the Euclidean separation can at times be deceiving. Figure 2 appeared underneath outlines this with a case of the width and tallness estimations of a question. Regardless of the two estimations being taken in the same physical units, an educated choice must be made with regards to the relative scaling. As the Figure 3 appears, changed scaling can prompt diverse clusterings.

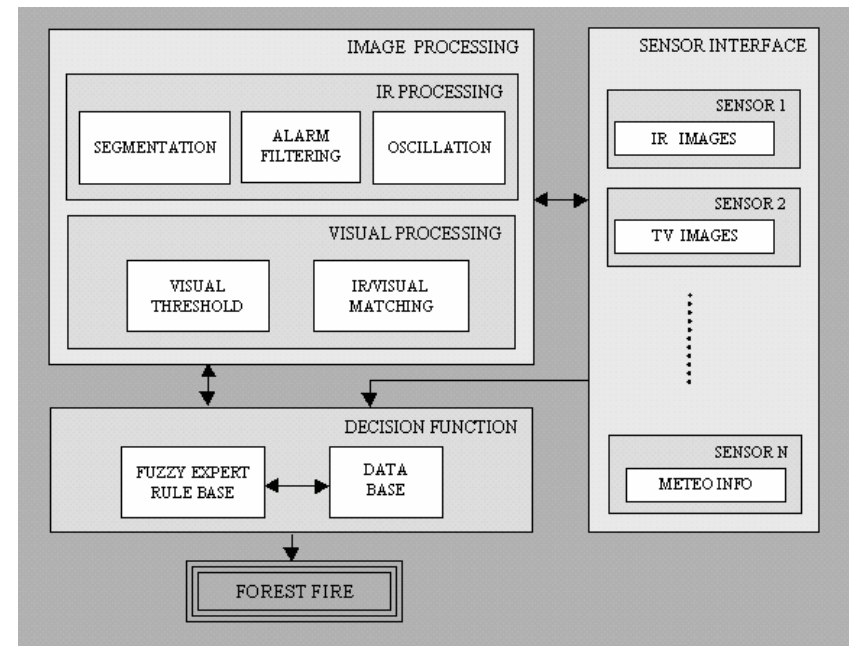

Figure 1. Existing block diagram. 


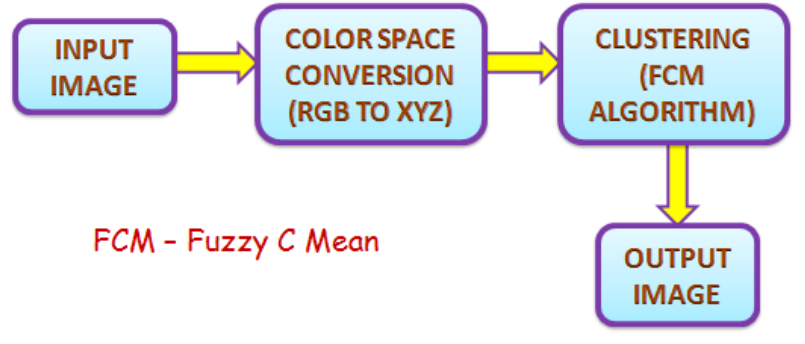

Figure 2. Proposed block diagram.

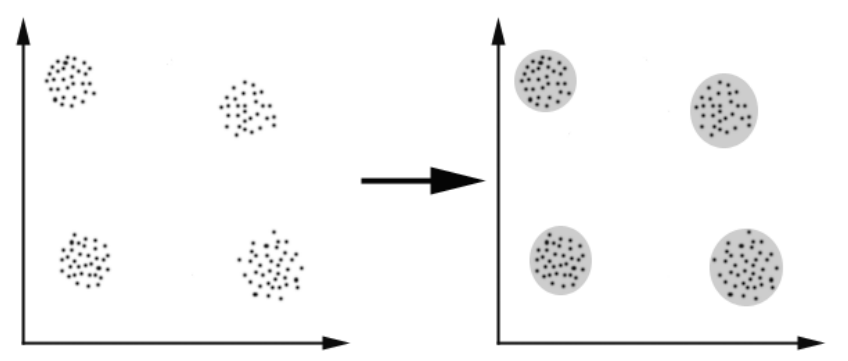

Figure 3. Distance Measure.

\subsection{Fuzzy C-Means Clustering Algorithm}

This calculation works by allotting enrollment to every datum guide relating toward each group fixate based on remove between the bunch focus and the information point. Increasingly the information is close to the group focus more is its enrollment towards the specific bunch focus. Plainly, summation of enrollment of every datum point ought to be equivalent to one. After every cycle enrollment and bunch focuses are refreshed by the recipe as shown in Figure 4.

Notice however this isn't just a realistic issue: The issue emerges from the numerical equation used to join the separations between the single parts of the information include vectors into a one of a kind separation measure that can be utilized for bunching purposes: Distinctive recipes prompts diverse clusterings. Once more, area learning must be utilized to manage the definition of an appropriate separation measure for every specific application. Gives best outcome for covered informational collection and similarly better then k-implies calculation. Not at all like k-implies ${ }^{5}$ where information point should only have a place with one group focus here information point is allotted participation to each bunch focus because of which information point may have a place with in excess of one group focus. It has a few disservices like An earlier

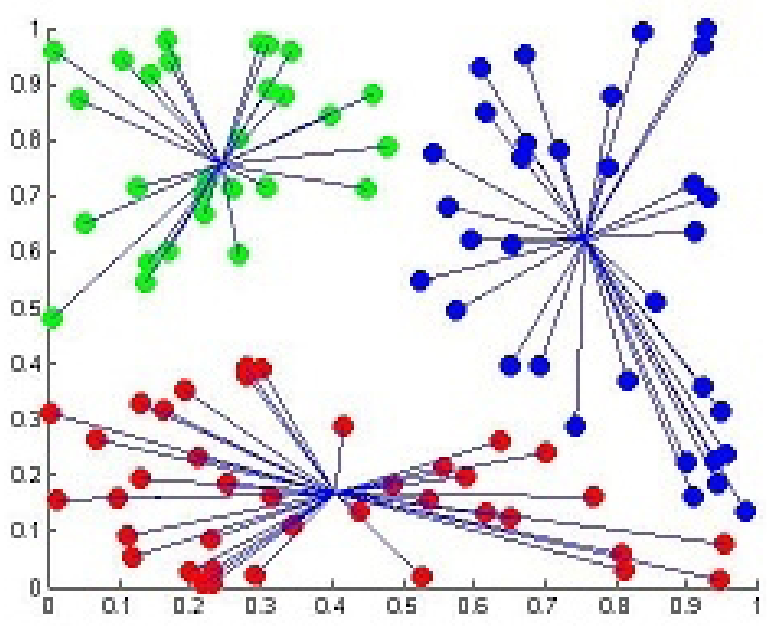

Figure 4. Result of Fuzzy C-means clustering.

particular of the quantity of groups and With bring down estimation of $\beta$ we show signs of improvement result yet to the detriment of more number of emphasis, Euclidean separation measures can unequally weight basic variables.

\section{Forest Fire Detection}

There is a two way to occur fire in modern days. One Natural and other Man made. There are all due to Combustion of dry fuel such as sawdust leaves, Smoking, recreation and equipment ${ }^{-6}$. Problem - Heat, Oxygen, fuel $=$ fire triangle. Fires are classified in to 1 . Smoldering fire that emits smoke but no flame and rarely self sustained. 2. Flaming combustion when flames present. 3. Charcoal can be formed-absence of $\mathrm{O}_{2}$. 4. Glowing combustionlater stage-blue flame. Types of fires are Ground fires occur on ground below leave in Figure 5. Surface fires on surface of forest above 1.3 meter high in Figure 6 and Crown fires-most danger-spread faster occurs top of trees in Figure 7. The safety measures are Ground fire: Digging trenches in soil layer, Surface fire: Portable water back pacts, Crown fire: Aerial support

\subsection{Mapping and Monitoring}

Global Positioning systems are used to monitoring the fire. It is a worldwide radio navigation system formed from constellation of 24 satellites and their ground stations. It utilizes these satellites as reference focuses to figure positions precise to matter of meters. GPS receivers $^{\underline{6}}$ are small in cars, boat, planes, constant equipment and laptop. 


\subsection{Color Conversion}

Shading is the brains response to a particular visual boost. Despite the fact that we can precisely portray shading by estimating its unearthly power circulation this prompts a vast level of redundancy. The reason for this redundancy is that the eye's retina tests shading utilizing only three expansive groups, roughly relating to red, green and blue light ${ }^{z}$. The signs from these shading delicate cells, together with those from the poles are consolidated in the mind to give a few unique "sensations" of the shading.

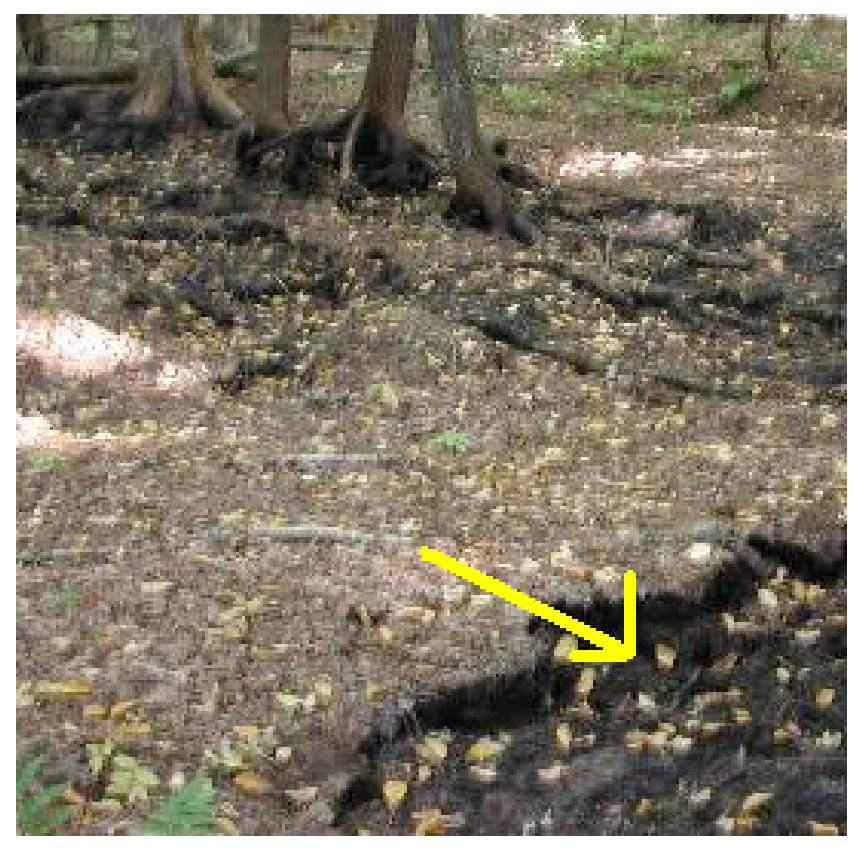

Figure 5. Ground fire.

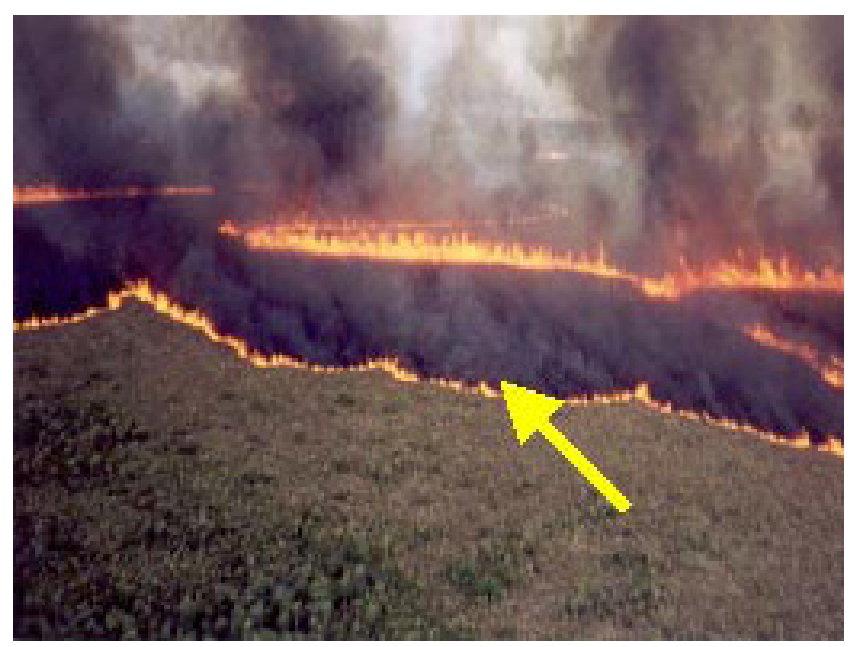

Figure 6. Surface fire.

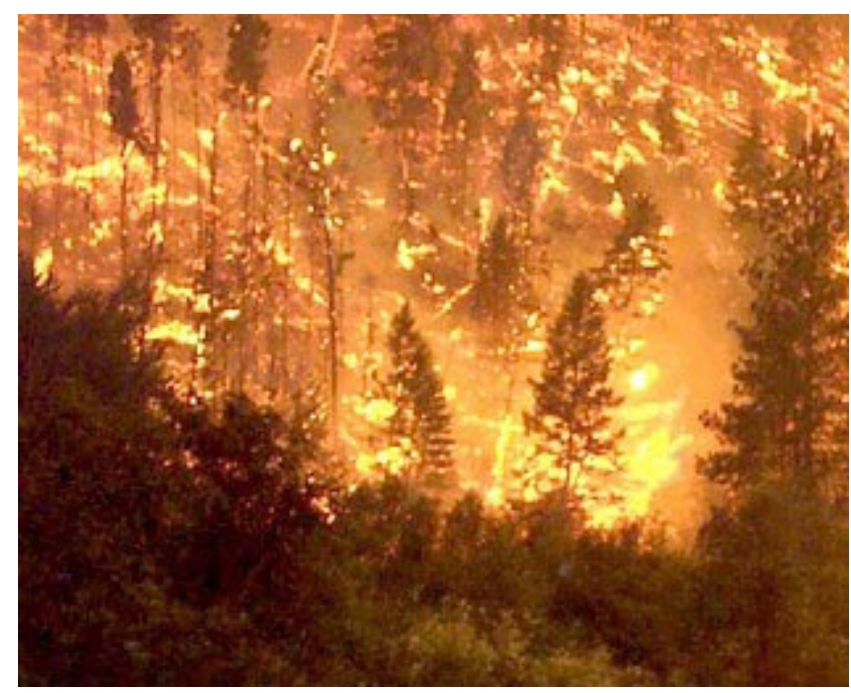

Figure 7. Crown fire.

\subsection{CIE XYZ}

The CIE XYZ system is at the foundation of all colorimetry. It is characterized with the end goal that every noticeable shading can be characterized utilizing only positive esteems and, the Y esteem is luminance. Consequently, the shades of the XYZ primaries themselves are not noticeable. The chromaticity chart is highly non-direct, in that a vector of unit size speaking to the distinction between two chromaticity's isn't uniformly obvious. A shading characterized in this system is alluded to as XYZ. A third co-ordinate, $z$, can likewise be characterized yet is repetitive since $\mathrm{x}+\mathrm{y}+\mathrm{z}=1$ for all hues.

\subsection{CIE XYZ Color Model}

The XYZ shading space is a worldwide standard created by the CIE. This model depends on three hypothetical primaries ${ }^{\underline{Z}}, \mathrm{XYZ}$ and every single obvious shading can be spoken to by utilizing only positive estimations of $X$, $\mathrm{Y}$ and $\mathrm{Z}$. The CIE XYZ primaries are hypothetical since they don't compare to any genuine light wavelengths. The $\mathrm{Y}$ primary is intentionally characterized to coordinate closely to luminance, while $\mathrm{X}$ and $\mathrm{Z}$ primaries give shading data. The primary favorable position of the CIE XYZ space (and any shading space in view of it) is that this space is completely gadget autonomous.

\subsection{Clustering}

A technique in which extraordinary, enrolled, covering, rasters are diminished to a singular raster, which is 
suggested as a cluster layout. The rasters used as a piece of grouping speak to investigative data. Particular bundling procedures use fluctuating method of reasoning to accomplish this mapping and dimensional reducing. At the point when all is said in done, most of the systems use testing different regards for each cell against all other cell regards to make sense of which subpopulation each cell is most like and should be accumulated with. All grouping methods first make a preparatory game plan of social affairs by testing all telephones or a case of them. They by then proceed by various procedures to test particular cells and progressively rethink the clusters until the point that the moment that each telephone is acceptably named a gathering interest in a singular pack, which is recorded at that telephone's circumstance in the bundle layout. The greatest number of groups to shape and the amount of refinement cycles is typically controlled in the midst of setup.

\subsubsection{Cluster Labeling}

Making sense of and gathering the bunches in the group outline thing that outcome from a programmed photograph understanding. The individual picks marks (names for styles of capacities) fundamentally in view of his or her data of the districts or materials inside the photo.

\subsubsection{Cluster Map}

The yield raster made by bunching or through unsupervised class ${ }^{z}$. The bunches are generally distinguished or arranged as some helpful type of material (as an occasion, a rural yield, a casing tissue kind or a dirt sort). It's far urgent to watch that this raster does not include values that can be subjected to comparably scientific investigation because of the reality the groups and their reidentification as texture or place sorts do now not constitute information esteems which may be scientifically relentless. For instance, cells in a group self-assertively appointed the estimation ${ }^{-8}$ of 4 through the bunching strategy does never again dependably constitute twice as a lot of something as a cell relegated to a group that changed into given a charge of 2 .

\subsubsection{Labeling}

Recognizing and gathering the bunches that outcome from any kind of automated photograph understanding.
You select principally in view of your know-how of the districts or materials inside the picture.

\section{Experimental Results}

Here the below images shows that input images and their results.Figure 8 is the input image which is used to detect the forest fire. The consecutive images are in Figures 9-11 are converted from RGB in to XYZ. Based on the results those images are going too segmented by using clustering methods. Here we are using $\mathrm{K}$ means algorithm for clustering. One of the clustered imagesis selected based on the visibility and information having. Finally by using merging and mapping method we can identify the forest fire area in image by using algorithm

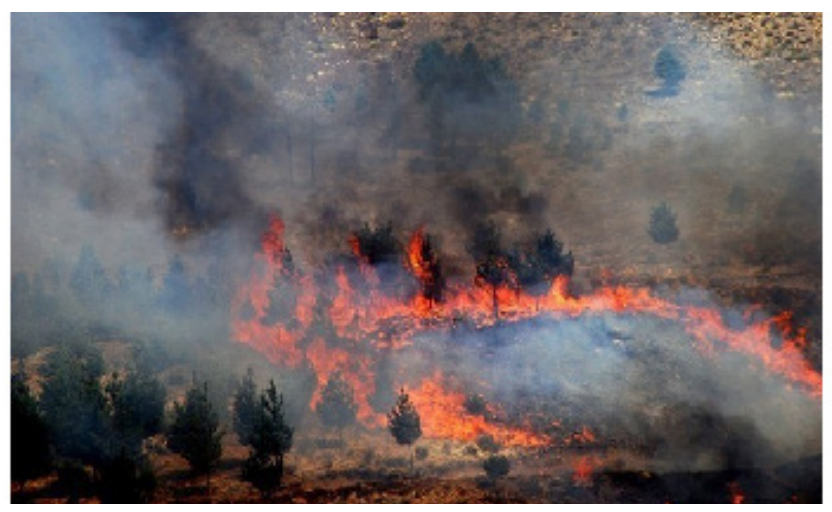

Figure 8. Input image.

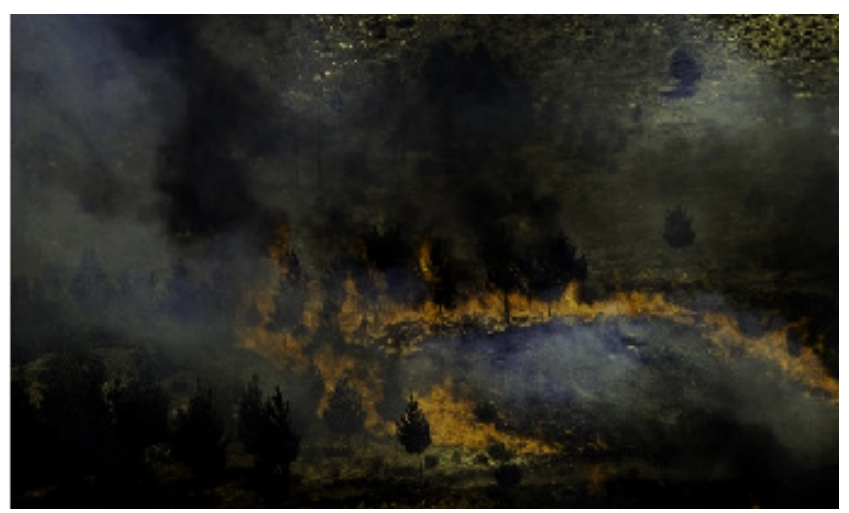

Figure 9. RGB to XYZ image. 


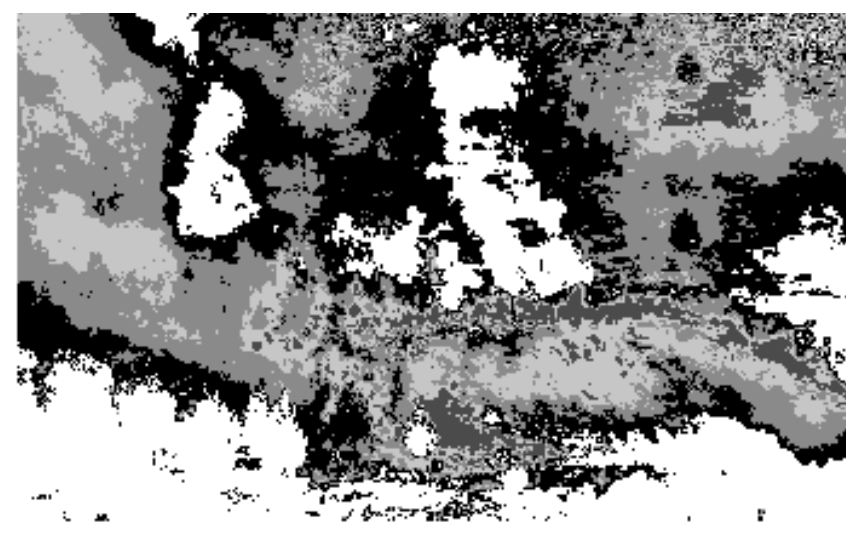

Figure 10. Clustered image.

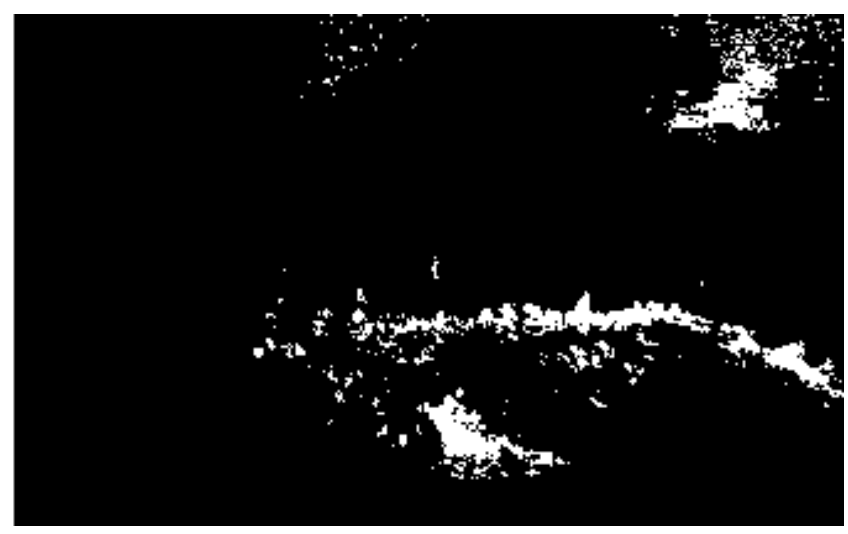

Figure 11. Fire detected region.

\section{Conclusion}

Here spatial data is used to locate the regions which susceptible to forest fire with the help of Image processing. Also Fuzzy C Mean algorithm is used to design and locating fire areas in images. Here images are clustered using
FCM algorithm which locates the regions of fire. Then the XYZ color method values of pixels which are clustered for detecting the presence of fires. This system is designed and produces the effective result for the people in surveillance of fire areas.

\section{References}

1. Ghosh S, Dubey SK. Comparative analysis of K-means and fuzzy C-means algorithms. International Journal of Advanced Computer Science and Applications. 2013; 4(4):35-9. Crossref.

2. MaheswariS, Korah R. Review on image segmentation based on color space and its hybrid. Proceedings in International Conference on Control, Instrumentation, Communication and Computational Technologies; 2017. p. 639-41.

3. Zhang J, Li W, Yin ZX. Forest fire detection system based on Wireless Sensor Network. Proceedings in 4th IEEE Conference on Industrial Electronics and Applications; 2009. p. 520-3. Crossref.

4. Bhowmik S, Datta V. A survey on clustering based image segmentation. IJARCET. 2012 Jul; 5(1):280-4.

5. Ichihashi H, Miyagishi K, Honda K. Fuzzy C-means clustering with regularization by $\mathrm{KL}$ information. Proceedingsin10th IEEE International Conference on Fuzzy Systems; 2001. p. 924-7. PMCid: PMC87266.

6. Gharieb RR, Gendy G. Fuzzy C-means with a local membership KL distance for medical image segmentation. Proceedings in IEEE Cairo International Biomedical Engineering Conference (CIBEC); 2014. p. 47-50. Crossref.

7. Rathore VS, Kumar MS, Verma A. Colour based image segmentation using $L^{\star} A^{\star} B^{\star}$ colour space based on genetic algorithm. International Journal of Emerging Technology and Advanced Engineering. 2012 Jun; 2(6):156-62.

8. Mythili C, Kavitha V. Color image segmentation using ERKFCM. International Journal of Computer Applications. 2012 Mar; 41(20):21-8. https://doi.org/10.5120/58098074 